\title{
Study of micro-trichome (mict) reveals novel connections between transcriptional regulation of multicellular trichome development and specific metabolism in cucumber
}

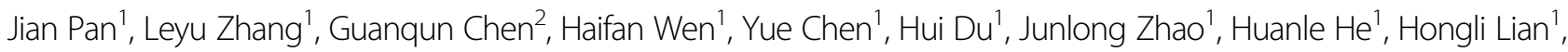 \\ Huiming Chen ${ }^{3}$, Jianxin Shi ${ }^{4}$, Run Cai ${ }^{1,5}$, Gang Wang ${ }^{1}$ and Junsong Pan $\mathbb{D}^{1}$
}

\begin{abstract}
Trichomes that cover the epidermis of aerial plant organs play multiple roles in plant protection. Compared with a unicellular trichome in model plants, the development mechanism of the multicellular trichome is largely unclear. Notably, variations in trichome development are often accompanied by defects in the biosynthesis of cuticle and secondary metabolites; however, major questions about the interactions between developmental differences in trichomes and defects in metabolic pathways remain unanswered. Here, we characterized the glabrous mutant mict/ csg/1/cstbh via combined metabolomic and transcriptomic analyses to extend our limited knowledge regarding multicellular trichome development and metabolism in cucumber. Mict was found to be explicitly expressed within trichome cells. Transcriptomic analysis indicated that genes involved in flavonoid and cuticle metabolism are significantly downregulated in mict mutants. Further metabolomic analysis confirmed that flavonoids, lipids, and cuticle compositions are dramatically altered in mict mutants. Additional studies revealed that Mict regulates flavonoid, lipid, and cuticle biosynthesis by likely directly binding to downstream functional genes, such as CsTT4, CSFLS1, CsCER26, and CSMYB36. These findings suggest that specific metabolic pathways (e.g., flavonoids and cuticle components) are co-regulated by Mict and provide insights into transcriptional regulation mechanisms of multicellular trichome development and its specific metabolism in cucumber.
\end{abstract}

\section{Introduction}

Plant trichomes are highly specialized structures located on the surfaces of aerial organs. They play essential roles in diverse biotic and abiotic adaptation, for instance, deterring insects, herbivores, and microbes; secreting ions and contaminated metals $^{1}$; reducing mechanical wear; regulating surface temperature; collecting and dispersing pollen; and absorbing water and

Correspondence: Gang Wang (wg770801@sjtu.edu.cn) or

Junsong Pan (jspan71@sjtu.edu.cn)

'School of Agriculture and Biology, Shanghai Jiao Tong University, Shanghai 200240, China

${ }^{2}$ School of Design, Shanghai Jiao Tong University, Shanghai 200240, China

Full list of author information is available at the end of the article

These authors contributed equally: Jian Pan, Leyu Zhang nutrients ${ }^{2}$. Morphologically, trichomes can be glandular trichomes or non-glandular trichomes, unicellular or multicellular, and branched or unbranched. In addition, they have become an excellent model system for the analysis of cell differentiation as they are easily accessible in model plants such as Arabidopsis thaliana ${ }^{3}$. Trichome development was reported to have a close relationship with increased metabolite levels, such as flavonoids, anthocyanins, and phenylpropanoids ${ }^{4}$.

In the model plant Arabidopsis with non-glandular and unicellular trichomes, expression maps of leaf trichomes revealed high anthocyanin and flavonoid pathway activities, indicating the roles of trichomes in secondary metabolism and defense ${ }^{4}$. Similarly, in Brassica rapa with

\section{(c) The Author(s) 2021}

(c) (i) Open Access This article is licensed under a Creative Commons Attribution 4.0 International License, which permits use, sharing, adaptation, distribution and reproduction cc) in any medium or format, as long as you give appropriate credit to the original author(s) and the source, provide a link to the Creative Commons license, and indicate if changes were made. The images or other third party material in this article are included in the article's Creative Commons license, unless indicated otherwise in a credit line to the material. If material is not included in the article's Creative Commons license and your intended use is not permitted by statutory regulation or exceeds the permitted use, you will need to obtain permission directly from the copyright holder. To view a copy of this license, visit http://creativecommons.org/licenses/by/4.0/. 
non-glandular trichomes, overexpression of BraLTP2 leads to increased trichome density and affects the accumulation of secondary metabolites ${ }^{5}$. In Solanum lycopersicum L., jasmonic acid-insensitive1 (jai1) is defective in JA signaling, and there was a significant reduction in the density and type of trichomes and monoterpene levels in the jai-1 mutant ${ }^{6}$. In addition, Trichomes in the hairless $(h l)$ mutant show abnormal morphology and density, reduced sesquiterpene and polyphenolic compounds, and increased susceptibility to insects and pathogens ${ }^{7}$. In addition, $S l C y c B 2$, which encodes a B-type cyclin gene, is involved in the initiation of multicellular trichomes and terpenoid biosynthesis. Nevertheless, little is known about the developmental process and underlying regulatory molecular mechanisms of multicellular non-glandular trichomes.

Cuticles are composed of polymer matrix cutin that is covered and embedded with waxes, and both are synthesized by underlying epidermal cells. Cutin is a biopolyester composed of C16 and C18 hydroxy and epoxy fatty acids, while wax is a complex mixture of very long chain fatty acids (VLCFAs), predominantly of chain lengths from 26 to 34 carbons. Nevertheless, wax also includes secondary metabolites and triterpenoids, such as flavonoids and sterols ${ }^{8}$. Wax aliphatic compounds are produced via the alcohol-forming and alkane-forming pathways ${ }^{9}$, leading to VLC alkanes and their derivatives ${ }^{10}$. eceriferum (cer) mutants present abnormal wax compositions or constituents in Arabidopsis thaliana ${ }^{11}$. According to mutant phenotype expression patterns and heterologous expression in yeast, CER6 participates in producing fatty acids with 26 and 28 carbons $^{12}$. Notably, it has been proven that CER26 has a major role in C30 elongation, with high tissue and substrate specificity ${ }^{13}$.

Cuticles are also special structures on the epidermis of aerial plant organs. However, different from trichomes, which are generally considered "biofactories" for producing valuable natural products, the cuticle is the interphase between the plant and its surrounding environment ${ }^{14}$. In Artemisia annua, a novel HD-ZIP IV/ MIXTA complex plays a significant role in regulating epidermal development, including cuticle formation and glandular trichome initiation ${ }^{15}$.

In cucumber, trichomes are multicellular, unbranched cells that are regularly distributed on most aerial surfaces. tril/csgl3 and csgl1/mict are important cucumber mutants that enable the dissection of trichome development into distinct, genetically controlled steps, as follows: Trichomeless (Tril) regulates the initiation and density of the trichome and Micro-trichome (Mict) allows trichomes to differentiate correctly ${ }^{16,17}$. Both Tril and Mict are HD-ZIP transcription factors, different from the well-known MYBWD40-bHLH complex in Arabidopsis. Unlike the unicellular trichomes in Arabidopsis, trichomes in cucumber allow cells to divide and to form into specialized trichome cells, such as fruit spines and fruit tumors. The regulatory network for the formation of trichomes and its relevance to cuticles in cucumber remains largely unknown.

In a previous study, we found that Mict regulates trichome initiation ${ }^{17}$. In this study, we investigated the underlying metabolomic and transcriptomic changes in mict mutants and uncovered Mict regulatory pathways in the cuticle and secondary metabolism. The results provide valuable molecular information for further studies on cucumber fruit external quality traits related to market value.

\section{Results}

Mict loss of function mutants exhibit profoundly altered leaf and fruit epidermis properties

Mict has previously been reported to be involved in the development of trichomes in cucumber fruits and leaves ${ }^{17}$. However, many other profound phenotypic changes were found in mict mutants; for example, mutant leaves looked brighter and glossier (Fig. 1a, b) and mutant fruit surfaces were smooth (Fig. 1c). However, the mict mutant sarcocarp did not show visible phenotypic differences in color, gloss, or cell morphology compared with wild-type (Fig. 1d). Gloss levels (gloss unit value) of the leaves and fruits were examined by a gloss meter, which showed that mict leaf but not fruit surfaces were glossier than those of the WT (Fig. 1e). The gloss levels of the mict leaves were significantly higher than those of WT leaves even when trichomes were removed artificially, indicating that the gloss unit value is not affected by the presence or absence of trichomes. This result implied that mutation of Mict affects not only epidermal properties, but also epidermal cell metabolism in cucumber.

\section{Mict expression is highly associated with trichome development}

To understand the in-depth function of Mict in the development of trichomes, comparative phenotypic observations of early developmental stages of trichomes were carried out (Fig. 2a).

The most apparent differences in trichomes between the wild-type and mict mutant occurred from Stages III to $\mathrm{V}$, where the mutant trichomes maintained a bulge shape without cell division or elongation that occurred in the wild-type (Fig. 2a). Therefore, Mict regulates trichome development at early stages via its function in cell division or elongation.

We further examined the spatiotemporal expression pattern of Mict and revealed that Mict mRNA levels were dramatically elevated during the early trichome developmental stages, initiating from Stage I, sharply increasing at Stage II, and peaking at Stage IV while decreasing slightly 


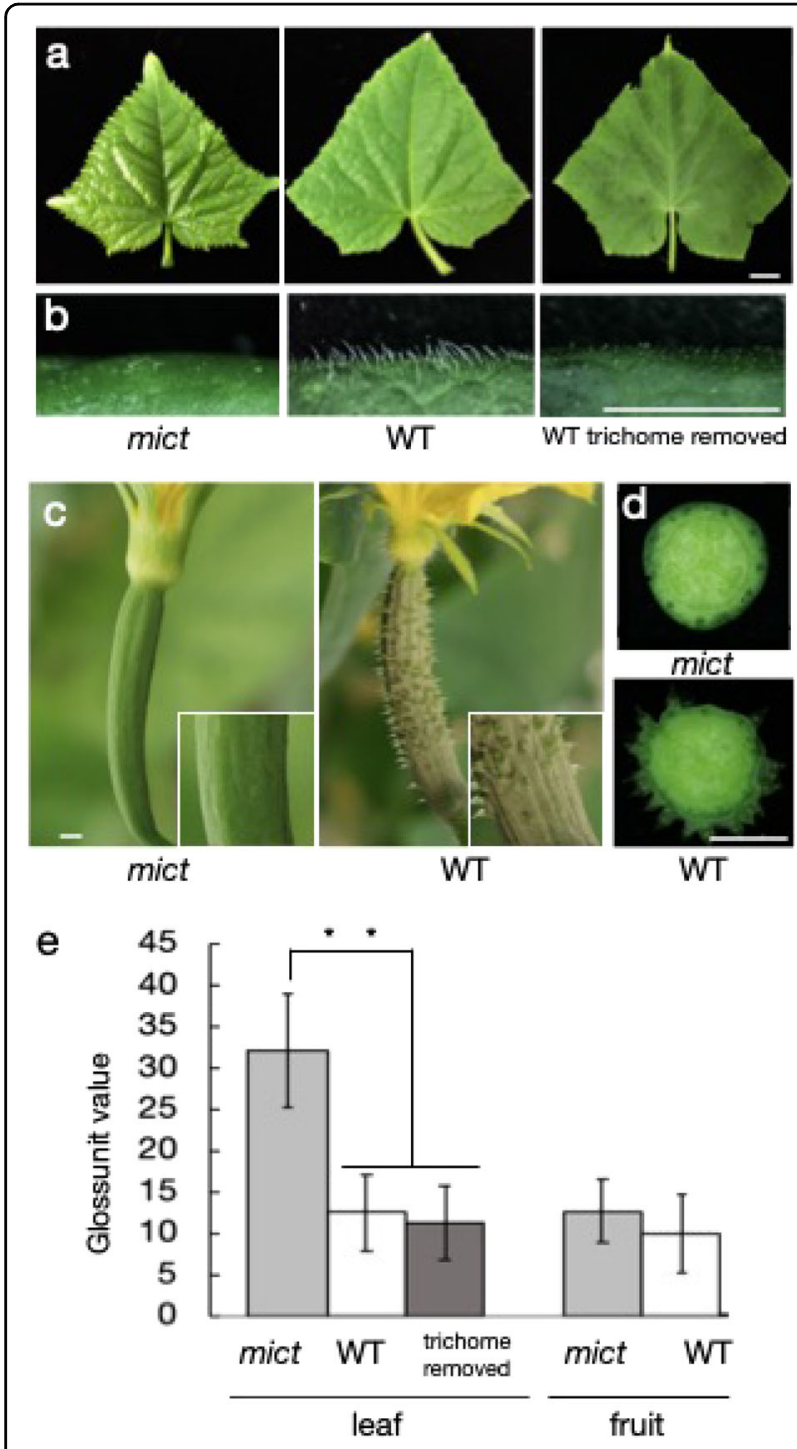

Fig. 1 Phenotypic analysis of mict mutants and wild-type cucumber plants. a Phenotype of mict mutant and wild-type (WT) leaves as well as WT leaves with trichomes removed (from left to right). $\mathbf{b}$ The leaf surfaces corresponding to (a). c Phenotype of mict mutant and WT fruits. $\mathbf{d}$ Cross-section of mict mutant and WT fruits. e Gloss unit value of leaves and fruits from the mict mutant and WT. The asterisks represent significant differences between samples (Student's $t$-test, ${ }^{* *} P<0.01, n=10$ )

in Stage V (Fig. 2b). Mict was highly expressed in the fruit spine and barely expressed in tissues without the spine (Fig. 2b). Noticeably, in situ hybridization showed that Mict transcripts were specifically detected in the first cell of the multicellular trichome (Fig. 2c-f), which indicated that Mict is involved in the early stage of trichome development, particularly in initiating the transformation of trichomes from single-cell bulges to cone-shaped multicellular trichomes.
Loss-of-function of Mict strongly affects global changes in genes involved in flavonoid pathways and cuticle metabolism

To reveal the global effects of Mict on leaf trichomes and fruit spine development, transcriptome analysis was performed to identify significantly changed metabolic pathways and putative downstream targets of Mict. For the RNA-seq of mict and wild-type plants, we collected expanded leaves at the four leaves seedling stage and fruits (the blooming female flower's ovary). FPKM values were used to calculate gene expression levels and differential expression was defined by statistical parameters $(P<0.05$ and fold change $>2$ or $<-2$ ). A total of 2548 (leaf) and 711 (fruit) genes exhibited differential expression, including 1367 (leaf) and 481 (fruit) upregulated genes and 1181 (leaf) and 230 (fruit) downregulated genes (Table S1).

An analysis of Kyoto Encyclopedia of Genes and Genomes (KEGG) pathways showed that differentially expressed genes (DEGs) were significantly enriched in flavonoids, fatty acids, and cuticle biosynthesis metabolic pathways in both the leaves and fruits (red asterisks in Fig. 3a, and Table S1). Venn analysis identified a common set of 359 DEGs between the leaves and fruits, and heatmap analysis showed that they showed consistent expression patterns between these two tissues (Fig. 3b, c). STRING analysis ${ }^{18}$ was used to predict the functional association networks of the homologs to Arabidopsis among these 359 DEGs, which uncovered two arrays of functionally related genes that were closely associated with epicuticular cuticle formation, including waxes, cutin, and flavonoids (Fig. 3d, e). This was in agreement with previous reports of the components of cuticles in plants ${ }^{8}$.

\section{Compositions of wax and cutin are largely changed in mict leaves and fruits}

Gas chromatography-mass spectrometry (GC-MS) analysis was carried out to characterize the chemical composition of the leaf and the enzymatically isolated cuticle from mict fruit. Overall, compared with wild-type, the abundance of cutin monomer was increased approximately 2 -folds in both the mict leaf and fruit. The significant increase in the amounts of fatty acids and midchain hydroxylated fatty acids in the leaf, and fatty acids and terminal hydroxylated fatty acids in the fruit mainly contributed to the accumulation of cutin monomers (Tables S2 and S3).

The total waxes in mict were reduced to $23.66 \%$ (leaf) and $53.33 \%$ (fruit) of the values in wild-type. The significant reductions in the amounts of alkanes contributed to reduced wax compounds in the mict leaf (Tables S4 and S5). The concentration of fatty acids, alcohols, and alkanes changed little in the mict fruit, and the reduction in total wax was mainly caused by the significant reduction in the concentrations of unidentified monomers. The cuticle 

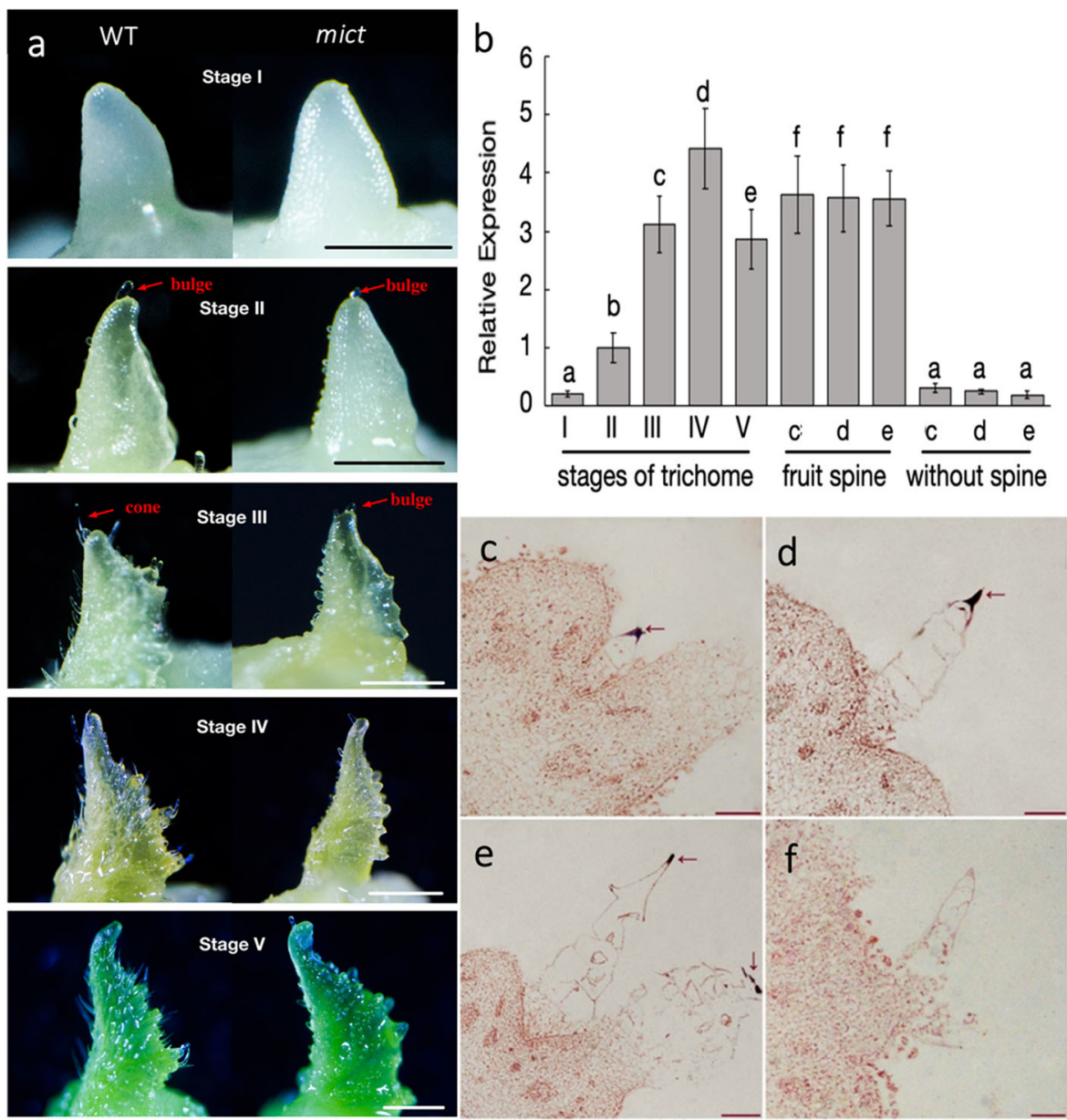

Fig. 2 Gene expression analysis of mict mutants and WT cucumber plants. a Trichome morphological observations in the 5 stages on the first leaf at $24 \mathrm{~h}, 32 \mathrm{~h}, 40 \mathrm{~h}, 48 \mathrm{~h}$, and $56 \mathrm{~h}$ after germination. $\mathbf{b}$ qRT-PCR assay of Mict expression in different organs. Panels (a-f) indicate significant differences by Tukey's multiple comparisons test with $P<0.05$ between different samples. (c-f) RNA in situ hybridization of Mict in WT, with (f) showing the sense-probe and (c-e) as the antisense-probe and fruits at $1 \mathrm{~cm}, 1.5 \mathrm{~cm}$, and $2 \mathrm{~cm}$. qRT-PCR results are shown in (b). Scale bars indicate $1 \mathrm{~mm}$ in (a) and $200 \mu \mathrm{m}$ in (c-f)

component differences in the leaf were more significant than in the fruit, which was consistent with the gloss measurements (Fig. 1e). These metabolic analyses clearly indicated that the loss-of-function of Mict also affects the leaf and fruit cuticle composition biosynthesis.

\section{The profiles of flavonoids and lipids are altered in mict leaves and fruits}

To further reveal the details of the metabolic changes, we performed a comparative metabolomic analysis in leaves and fruits of mict and wild-type plants using UHPLC-Q-TOF-MS. A total of 129 and 162 compounds with known structures were identified from the leaves and fruits, respectively. Among those, levels of 71 and 80 compounds were significantly different between the wild-type and mict mutant in leaves and fruits $(P<0.05)$, respectively (Table $\mathrm{S6}$ ).
The most notable metabolomic changes were observed in flavonoids and lipids (Fig. 4). In mict leaves, $70.37 \%$ and $14.81 \%$ of detected flavonoids were decreased and increased, respectively. In mict fruits, $80.4 \%$ and $15.22 \%$ of detected flavonoids were decreased and increased, respectively. For lipids, in mict leaves, $69.23 \%$ and $30.77 \%$ of detected lipids were decreased and increased, respectively. In mict fruits, $36.54 \%$ and $63.46 \%$ of detected lipids were decreased and increased, respectively.

This metabolomic results suggest that Mict significantly affects lipid and flavonoid metabolic pathways.

\section{Loss-of-function of Mict downregulates the expression of an array of genes involved in flavonoid and cuticle metabolism}

We performed a metabolite-gene correlation analysis to further identify the association of flavonoids and cuticle 


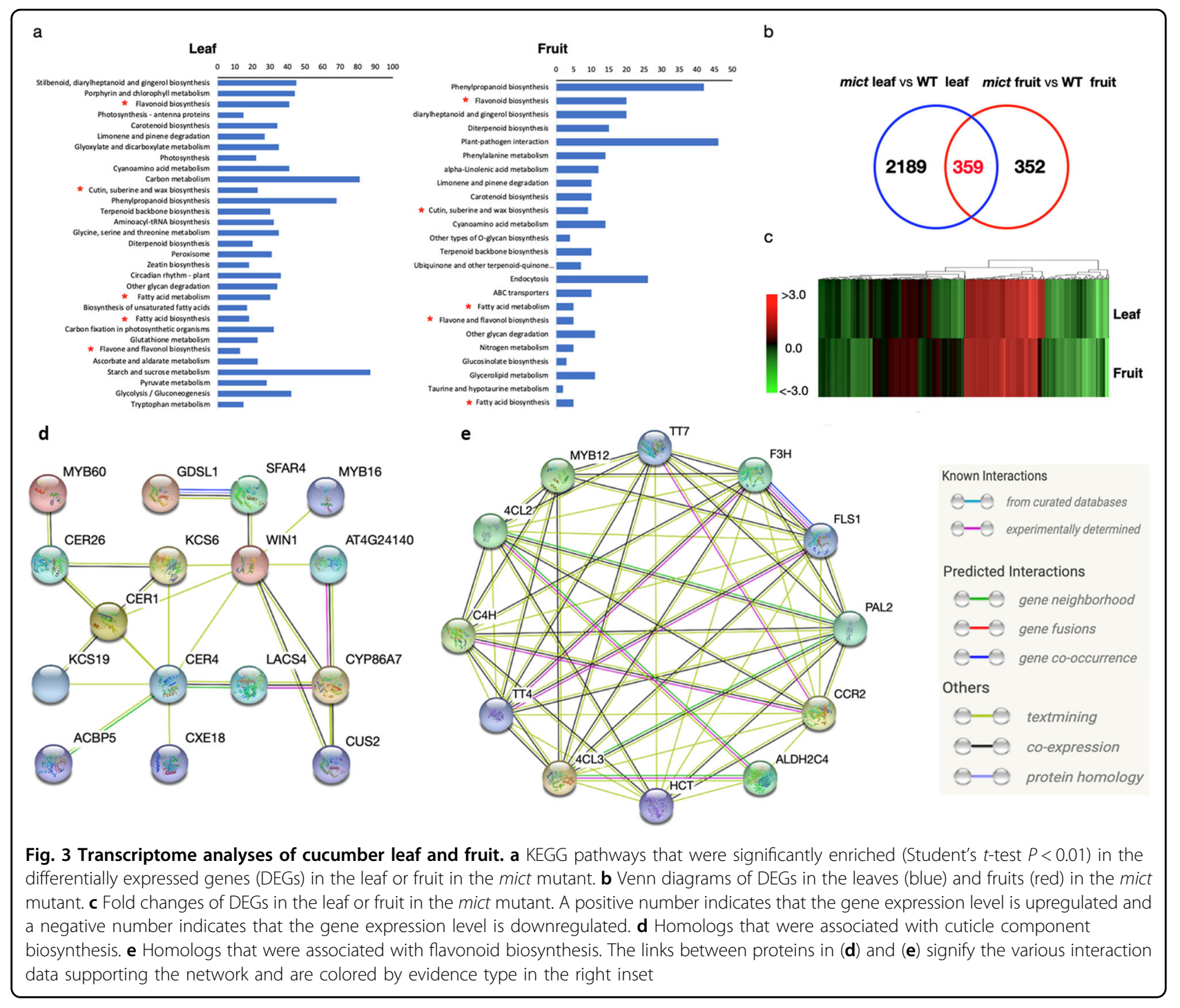

metabolism pathways with the putative Mict target genes. Among the DEGs, 15 putative cucumber orthologues of reported genes involved in cuticle formation in Arabidopsis showed significant downregulation in mict mutants (Fig. 5a). We further performed a qRT-PCR assay to test the expression levels of these genes in trichomes removed from leaves and fruit peels, which were in agreement with the cuticle composition analysis. We found that although expression of MAH1-like and LACS3 were not significantly changed, the other cuticle-related genes were downregulated in trichomes removed from mict leaves (Fig. S1). In fruit peel, except for CER6 and $H T H$, genes related to the cuticle were also downregulated in mict, which was nearly consistent with the transcriptome data (Fig. S1).

According to the metabolomic and transcriptomic results, flavonoid metabolism was largely hampered in the mict mutant, particularly at the step catalyzing $\mathrm{p}$ -
Cinnamoyl-CoA to kaempferol derivates (Fig. 6a). Moreover, the contents of naringenin chalcone, naringenin, and kaempferol derivates were substantially lower than that of the wild-type (Fig. 6c). Accordingly, the expression of the four key enzymes (i.e., TT4, CHI, F3H, and FLS1) that catalyze the above processes was repressed in the mict mutant. Other important genes, such as ANS, IFR, and $H C T$, were also downregulated to varying degrees in the mict mutant (Fig. 6b), implying that the flavonoid and flavone biosynthesis pathways are defective if Mict is dysfunctional.

\section{Mict positively regulates the expression of CsTT4, CsFLS1, CsCER26, and CSMYB36 by binding to their promoters}

Based on the multi-omic results that loss-of-function of Mict had a major impact on cuticle component metabolism (Fig. 4) and our finding that the downstream genes may contribute to the abnormal metabolic phenotype 

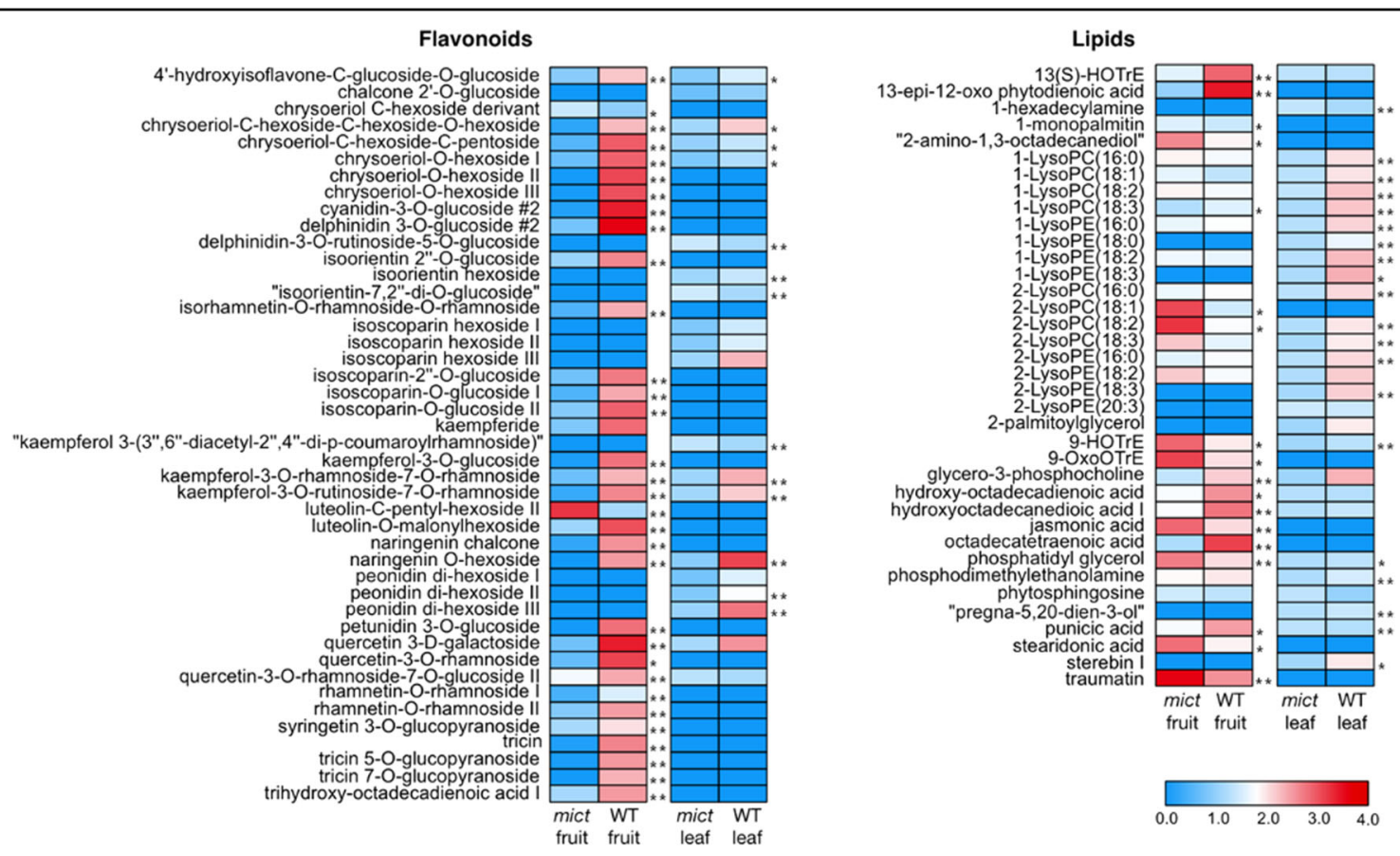

Fig. 4 Heatmap of metabolite changes in the fruits and leaves of WT (cucumber line 06-1) and mict mutant (06-2). Ratios of fold changes are given by shades of red or blue colors according to the scale bar. Ratios were calculated by taking the logarithm of the mean values from four biological replicates. Student's $t$-test: ${ }^{*} P<0.05 ;{ }^{* *} P<0.01$

\section{a}

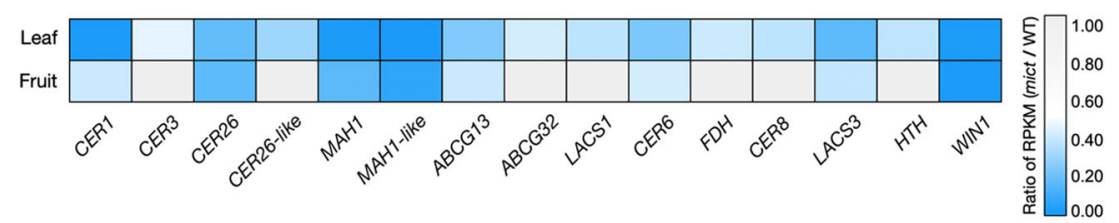

b

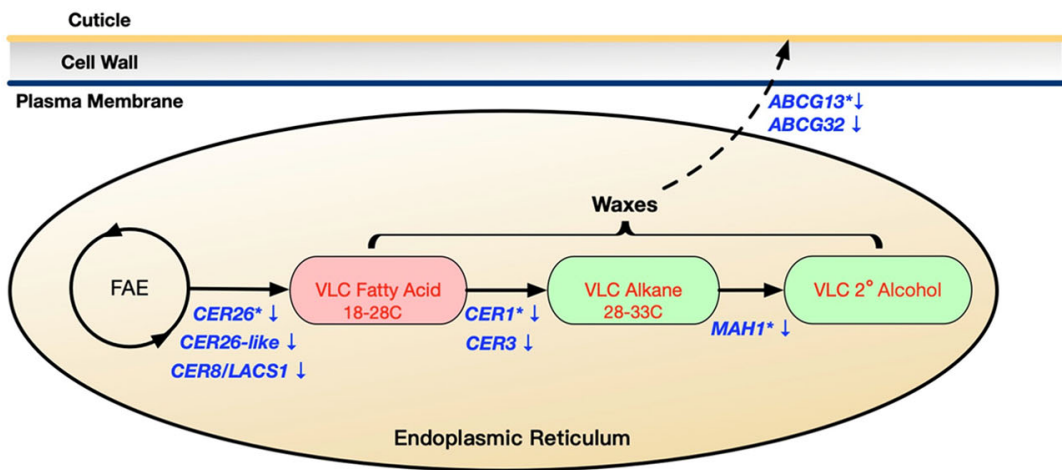

Fig. 5 Correlation analysis of the transcriptome and cuticle components. a DEGs involved in the cuticle-forming pathways in the leaf and fruit. b Simplified diagram of genes involved in cuticle biosynthesis. Genes (blue text) are described in the article. Red text denotes compound classes that are typically observed in wax components

(Figs. 5 and 6), we examined whether Mict could interact with the promoter of the revealed downstream target genes. We used a yeast one-hybrid assay to test the interaction between Mict and the promoters of 47 putative downstream target genes that are associated with the flavonoid and cuticle pathways (out of the 359 DEGs). The results showed that Mict could physically interact in vitro with four of these promoters (Fig. 7a), namely, CsTT4, CsFLS1, CsCER26 (Csa2G147920), and CsMYB36 (Csa2G352940). Meanwhile, co-infiltration of 35S:Mict 

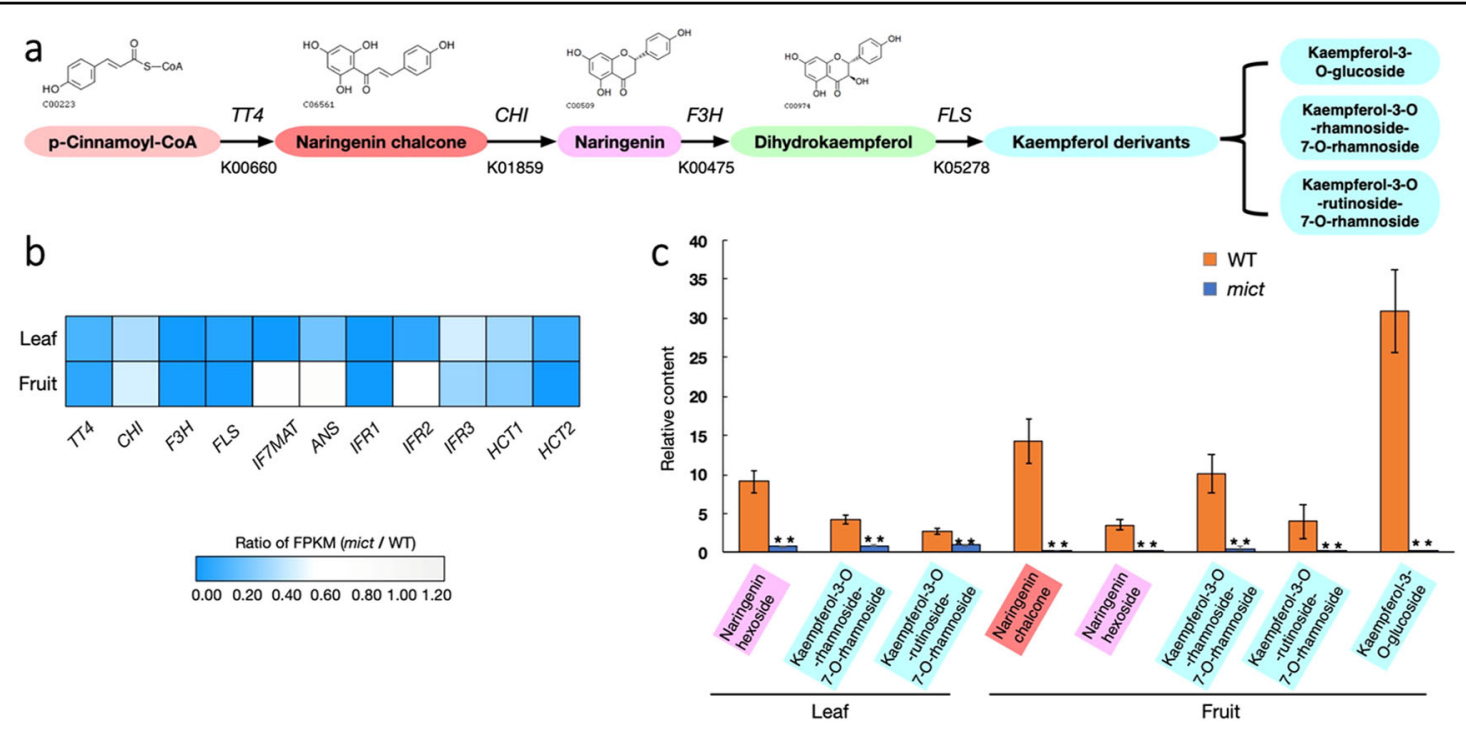

Fig. 6 Correlation analysis of transcriptome and flavone compounds. a Simplified diagram of part of the flavonoid biosynthetic pathway. TT4, transparent testa 4; CHI, chalcone isomerase; F3H, flavanone 3-hydroxylase; FLS, flavonol synthase. $\mathbf{b}$ DEGs involved in the flavone biosynthesis pathway in the leaf and fruit. c The relative abundance of metabolites related to the pathway in the leaf and fruit. The background color of the compound and its derivatives are consistent with (a). Error bars indicate SD $(n=3)$. Student's t-test: ${ }^{*} P<0.05 ;{ }^{* *} P<0.01$

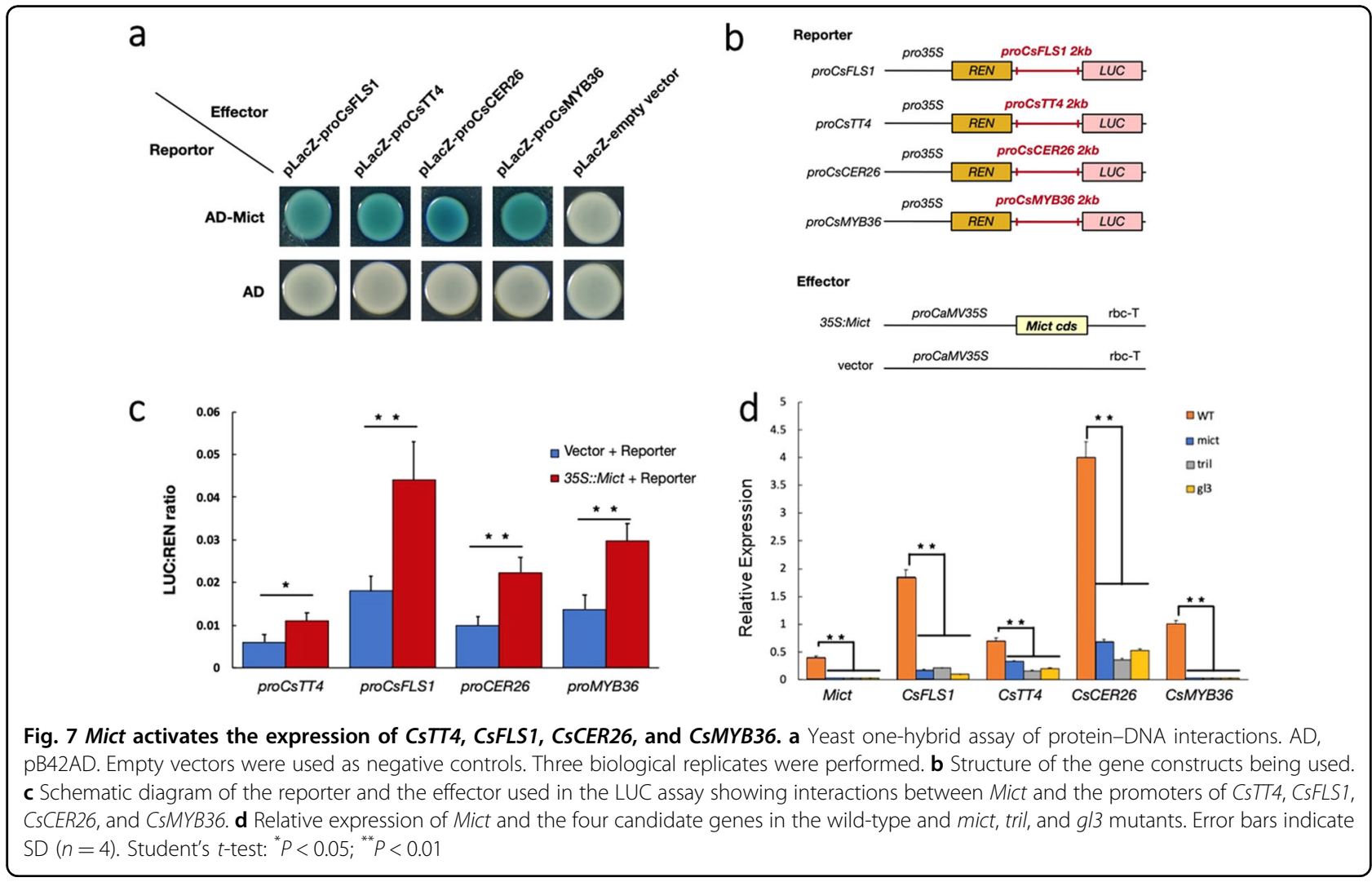

and promoter-LUC constructs resulted in significant transactivation of the promoters of the four tested putative Mict targets (Fig. 7b, c). To further explore the Mict binding region in the promoters, we segmented the promoters into four 500 bp long fragments (Fig. S3a). The results of yeast one-hybrid assay suggest that the CsFLS1, CsCER26, and CsMYB36 binding sites are located in the -2000 to -1500 bp regions of the promoter. For CsTT4, 
the binding site is located in the -500 to $0 \mathrm{bp}$ region in the promoter (Fig. S3b). In addition, these four genes were also significantly downregulated in the other two trichome mutants (trichome-less(tril) and glabrous 3(gl3)), which exhibit recessive epistasis toward mict and show a similar phenotype to the mict mutant with bright leaves and reduced wax upload (Fig. 7d).

To further confirm the biological functions of CsTT4, CsFLS1, and CsCER26, we created CsTT4, CsFLS1, and CsCER26 overexpression Arabidopsis transgenic lines. The naringenin chalcone and kaempferol-3-O-glucoside contents were significantly increased in the oxCsTT4 and oxCsFLS1 transgenic plants, respectively (Fig. S2a, b), which suggests that CsTT4 and CsFLS1 are functionally conserved in the flavonoid biosynthesis pathway. The CsCER26-overexpressing plants showed a glossy phenotype compared with the wild-type plant stem (Fig. S2c), which was consistent with the AtCER26-overexpressing Arabidopsis plants ${ }^{13}$. Taken together, these results suggest that Mict likely affects flavonoid and cuticle metabolism as a direct action on the gene expression of its target genes and is associated with epidermal cell development and patterning.

\section{Discussion}

Based on previous studies, the determination and morphogenesis of the multicellular trichomes of cucumber are regulated by HD-ZIP transcription factors ${ }^{5,15-17,19,20 \text {, }}$ which show differences from unicellular trichomes. The known genes related to the cucumber trichome (spine) development are tril, mict, and Tu (tuberculate fruit). Mict mediates the morphogenesis of trichomes, and disruption of Mict makes most trichomes remain in the single-cell bulge state. In this study, evidence from our multi-omic analysis shows that Mict acts as a regulator in the very beginning phase to turn on an array of biological processes related to trichome development, directly or indirectly. Although the functions of Mict target genes need to be tested in further research, our results indicate that Mict can activate genes involved in flavonoid and cuticular lipid biosynthesis. The main purpose of this study was to identify the connection between transcriptional regulation and metabolic pathways in trichome development via multi-omics analysis and provide evidence to support that Mict regulates metabolic pathways (e.g., flavonoids and cuticle) both directly and indirectly.

\section{Mict turns on the regulatory network at the beginning of trichome development}

Based on our spatial-temporal expression analysis results, Mict is highly expressed at Stage III and Stage IV and is reduced at Stage V, implying that Mict functions at a particular stage during trichome development in leaf. Moreover, transcription of Mict can hardly be detected in any other organs except fruit spines, which implies that Mict acts as a regulator in the very beginning of trichome initiation, modulating a large number of downstream target genes for a transient period. Then, the regulated genes continually promote processes, such as cell division and secondary metabolite biosynthesis, to promote proper trichome development. Other evidence that Mict acts as a turn-on switch is that Mict is accurately detected in the top cell of the multicellular trichome, inducing successive trichome cells to develop spontaneously after Mict activates the regulatory network in the top cell. The expression of transcription factors (e.g., CsWIN1, CsMYB6, and CsMYB36) is absent in mict mutants, which implies complex interactions between transcription factors are involved in the transcript regulatory network. As a result, the mict mutant shows a defective phenotype in wax biosynthesis. In this study, we narrowed the Mict binding sites into a $500 \mathrm{bp}$ region in the promoter of the four downstream genes. Common cis-element analysis of these four promoters suggested that only light response elements were identified in all four promoters (Fig. S3c). Taken together, Mict might bind novel elements in the promoters that specifically regulate trichome development, which merits further investigations.

\section{Mict regulates cuticle metabolism in epidermal cells}

The plant cuticle is an extracellular lipid structure deposited on the aerial surfaces of organs to protect the plant against environmental stresses ${ }^{21}$. Cuticles are complex mixtures of VLCFAs, with chain lengths of more than 26 carbons. In mutant leaf, the wax composition with more than 26 carbons changed significantly compared to that of WT. However, in mutant fruit, similar significant changes in wax components were lacking. Therefore, the change in VLCFA contents might contribute to observed phenotypes between the two organs. Transcriptomic study revealed significant DEGs involved in fatty acid and cuticle biosynthesis pathways, and led us to examine the cuticle's wax composition. In combination with metabolomics data, we constructed a schematic diagram to explain wax changes, such as VLCFAs, alkanes, and secondary alcohols, in mict mutants (Fig. 5b). Downregulation of CER26 and CER26-like indicated the elongation of 30 carbons or more was blocked in the mict mutant ${ }^{13}$. Downregulated CER8 in the mict mutant caused the lacing of alkanes and redundancy of free fatty acids ${ }^{22}$.

Moreover, low expression of $C E R 1$ and $C E R 3$, which act together to catalyze the formation of alkanes from VLCFA-CoA, also explains the lack of alkanes in the mict mutant ${ }^{23}$. MAH1, a midchain alkane hydroxylase that catalyzes secondary alcohol formation, was downregulated and related to the absence of $2-\mathrm{C} 18: \mathrm{OL}$ in the mict mutant $^{24}$. In addition, downregulated $A B C G 13$ and 
$A B C G 32$, functional $\mathrm{ABC}$ transporters involved in wax component transport, may also influence the transport of wax from the plasma membrane (PM) to the exterior of the cell ${ }^{25,26}$. In summary, the downregulated expression of the genes mentioned above could partly explain some of the compound changes in cuticle biosynthesis pathways, which means that Mict positively regulates the downstream genes directly or indirectly to control these pathways in cucumber leaves and fruits. These results are consistent with the mutation of genes related to trichomes, such as GL1, MYB16, MYB106, and CER6, which can also affect cuticle formation in Arabidopsis ${ }^{4,27-29}$.

Interestingly, a recent report found that the specific cuticular wax composition varies between different epidermal cell types, i.e., trichome and pavement cells ${ }^{30}$. However, in this context, it is remarkable that Mict has much stronger expression in trichome cells than in other types of cells, leading us to speculate that Mict regulates the specific cuticular wax components. Notably, CER26 has much stronger expression in trichome cells than in other epidermal cells in Arabidopsis ${ }^{13}$, making it reasonable that Mict positively regulates CsCER26 to synthesize the trichome-specific cuticular wax composition. It is technologically challenging for us to isolate multicellular trichomes from cucumber leaves, limiting an accurate examination of the trichome-specific cuticular wax composition. In the future, we believe that identification of the trichome-specific cuticular wax composition could reveal the function of Mict in cooperative regulation of trichome development and material metabolism.

\section{Mict regulates flavonoid metabolism in trichome development}

The trichome is regarded as a bio-factory of secondary metabolites in plants. The advantages of trichome-specific secondary metabolite biosynthesis could be maximizing the protective effects while minimizing the harm of their overaccumulation. Flavonoids are frequently used as pigments and involved in response to the environment and developmental processes ${ }^{31}$. Transcriptional control of the structural genes in the flavonoid biosynthetic pathway has been most extensively studied in plants. In our research, Mict was shown to regulate catalytic enzymes, such as CsTT4 and CsFLS1, which participate in flavonoid biosynthetic pathways. The expression of catalytic enzymes usually determines the content of metabolites as they are always located in the downstream regulation area. FLS is a key enzyme in flavonoid biosynthesis ${ }^{32}$, and TRANSPARENT TESTA (TT) family members were found to work as transcription factors or enzymes in flavonoid biosynthesis pathways in Arabidopsis. Among those, CHALCONE SYNTHASE (CHS), which is encoded by the TT4 gene, catalyzes the first step of flavonoid biosynthesis for producing naringenin chalcone, a common precursor of various flavonoids ${ }^{33}$. In addition, IF7MAT (isoflavone 7O-glucoside-6"-O-malonyltransferase), ANS (anthocyanidin synthase), $H C T$ (O-hydroxycinnamoyltransferase), and IFR (isoflavone reductase) also play key roles in flavone, flavonol, and flavonoid biosynthesis pathways. The aforementioned genes were greatly downregulated in mict mutants, suggesting they may participate in the trichomespecific secondary metabolite biosynthesis pathway via Mict-mediated regulation. Accordingly, together with the genes-metabolites association analysis in this study, Mict may act upstream in a way that activates regulatory networks in trichome morphogenesis and specific metabolic pathways, which ensures that trichomes develop correctly.

\section{Mict activates CsMYB36 to form fruit wax-powders}

The cucumber fruit wax-powder is a thin layer of white powder that surrounds the fruit, and it is mainly composed of VLCFAs (and its derivatives), such as alkanes, aldehydes, and primary and secondary alcohols ${ }^{34}$. Several cucumber mutants show no wax-powder on the fruits, such as csmyb36, tril/csgl3, and mict. It is clear that tril exhibits recessive epistasis with mict, as mict does to csmyb36, which suggests that CsMYB36 is the main regulator of wax-powder formation. In this study, we found that Mict could bind to the CsMYB36 promoter and stimulate its transcription. This result explains the absence of wax-powder on mict and tril fruits. Accordingly, the interaction between Mict and CsMYB36 directly bridges trichome development and wax-powder formation, and it provides us with a better understanding of the significance of trichome cell differentiation.

In this study, we identified the metabolic changes related to leaf trichome and fruit spine development in cucumber, and linked flavonoid and cuticle metabolism with transcriptional regulation. However, there are still many metabolites that we could not associate them with corresponding genes due to the limited cucumber molecular pathway databases. Fortunately, in recent years, with the rapid advances in trichome-related gene identification in cucumber, we believe our results will provide important clues to discover biochemical mechanisms in cucumber and reveal the molecular regulatory network involved in trichome development.

\section{Materials and methods \\ Plant materials}

The wild-type is cucumber (Cucumis sativus) North China type inbred line 06-1. The spontaneous mict mutant line 06-2 from 06-1 was grown under standard water, fertilizer management, and pest control with appropriate management in the glasshouse of Shanghai Jiao Tong University in Shanghai. Tobacco (Nicotiana benthamiana) and Arabidopsis were grown at $22^{\circ} \mathrm{C}$ in $24 \mathrm{~h}$ continuous light indoors. 


\section{Gloss levels measurement}

Fully expanded leaves and mature fruits of cucumber were samples for gloss levels measurement. The equipment we used is a Novo Curve Gloss Meter (RHOPOINT, England). Each measurement was repeated four times.

\section{Nucleic acid extraction and qRT-PCR}

Genomic DNA was extracted from the expanded leaves using the CTAB reagent. Fully expanded leaves and ovaries of female flowers were sampled on the day before flowering for RNA and were frozen in liquid nitrogen immediately and stored at $-80^{\circ} \mathrm{C}$. The RNA extraction, cDNA reverse transcription, and $\mathrm{qRT}-\mathrm{PCR}$ assays were performed as reported previously ${ }^{35}$. The gene-specific primers used for qRT-PCR were designed using Primer 3 software. Cucumber Actin3 and UBIQUITIN were used as internal controls to normalize and verify the expression data $^{36,37}$. Three biological replicates were used per gene. Each qRT-PCR experiment was performed with three technical replicates. The gene-specific primers are listed in Table S7.

\section{Materials for RNA-seq}

Total RNA was extracted from fully expanded leaves and ovaries of female flowers on the day before flowering. RNASeq for comparative transcriptomic analyses of the two phenotypes was performed with three biological replicates. The library was constructed and sequenced using a BGISEQ-500 by the Beijing Genomic Institution (BGI, China). The genomic DNA was removed with two digestions with amplification grade DNase I (Epigenetics, USA). Then, the material was treated as previously reported ${ }^{36,37}$. The clean data have been uploaded to the China National GeneBank (CNGB) (Project ID: CNS0230824, CNS0230826, CNS0230828, CNS0230830, CNS0230832, CNS0230834, CNS0230836, CNS0230838, CNS0230840, CNS0230842, CNS0230844, and CNS0230846).

\section{In situ hybridization}

Young cucumber fruits at different stages of growth $(1 \mathrm{~cm}, 1.5 \mathrm{~cm}$, and $2 \mathrm{~cm})$ were fixed with FAA. In situ sense and antisense probes were amplified with genespecific primers with SP6 and T7 RNA polymerasebinding sites, respectively. Sample fixation, embedding and pretreatment of sections, hybridization, and immunological detection were performed as described previously ${ }^{38}$. Primers of antisense and sense probes are given in Table S7.

\section{Bioinformatic analysis}

We conducted transcriptome profiling experiments using the DGE approach ${ }^{39}$. Samples were collected from the ovaries of female flowers on the day of flowering and fully expanded leaves from the wild-type and mict mutant.
Venn tools (Oliveros, 2007-2015) was used for Venn diagram analysis. To identify the homolog in Arabidopsis, we used the cucumber protein IDs $^{40}$ to batch query the Arabidopsis proteins (TAIR10) using BLASTP on the Cucurbit Genomics Database (http://cucurbitgenomics. org) with an e-value cut-off of $1 e^{-1}$. Based on the cucumber homolog in Arabidopsis, we predicted the protein interaction network using STRING (https:// string-db.org).

\section{Yeast one-hybrid assay}

For the yeast one-hybrid assay, the Mict open reading frames (ORFs) were amplified from 06-1 genomic DNA and then cloned into the $\mathrm{pB} 42 \mathrm{AD}$ vector (digestion with the EcoRI and XhoI restriction enzymes) using ClonExpress II (Vazyme, Nanjing, China). The $2 \mathrm{~kb}$ promoter of CsTT4, CsFLS1, CsCER26, and CsMYB36 from 06-1 were inserted into the vector (digestion with the EcoRI and Xhol restriction enzymes). pB42AD-Mict and placZCsTT4Pro, placZ-CsFLS1Pro, placZ-CsCER26Pro, or placZ-CsMYB36Pro were co-transformed into the yeast strain EGY48a. The empty vectors were used as negative controls. These transformants were cultivated to confirm whether Mict can bind the promoter of the CsTT4, CsFLS1, CsCER26, and CsMYB36 ${ }^{41}$. All of the primers used in this study are listed in Table S7.

\section{Dual-luciferase assay}

The assay was carried out as described previously ${ }^{20}$. Briefly, Mict ORFs were cloned into pHB driven by the $2 * 35 \mathrm{~S}$ promoter to obtain overexpression vectors. Then, the vectors were separately transformed into Agrobacterium tumefaciens GV3101 as effectors. The promoters of CsMYB36, CsFLS1, CsTT4, and CsCER26 from 06-1 were cloned into pGREEN 0800 to drive the firefly luciferase reporter gene. The CsMYB36, CsFLS1, CsTT4, and CsCER26 promoter vectors were then co-transformed as described previously ${ }^{35}$. The primers used in this study are listed in Table S7.

\section{Ectopic expression of CsTT4, CsFLS1, and CSCER26 in Arabidopsis}

To generate lines overexpressing CsTT4, CsFLS1, and CsCER26, the full-length coding regions of these three genes were amplified using specific primers containing the BamHI ( $5^{\prime}$ end) and SacI ( $3^{\prime}$ end) endonuclease sites, and inserted in the reverse orientation into the $\mathrm{pHB}$ vector. The recombinant plasmids were transformed into Col (WT) using the floral dip method ${ }^{42}$.

\section{Metabolite extraction and profiling}

The fully expanded leaves and ovaries of female flowers on the flowering day from the wild-type and mict mutant were ground into a fine powder. Twenty milligram of fine 
powder was used for metabolite extraction prior to UHPLC-Q-TOF-MS analysis. As the compound content was calculated by weight, the trichomes and fruit spines were not removed from the samples, which was similar to the transcriptome analysis. The metabolite extraction was analyzed as previously described ${ }^{43}$. All measurements were performed with three replicates.

\section{Analysis of cuticular wax and cutin chemical analysis}

As the compound content is calculated from the surface area, we removed the trichomes and fruit spines from the samples to reduce the area errors caused by the trichomes. For the fruit peels, we collected the peels from fruits at the 10th day after flowering so that the fruits were elongated enough that we could isolate the peel from the trichomes. Cucumber cuticular wax was extracted from fully expanded leaves and the fruit pericarp on the 10th day after flowering. Three independent wild-type (WT) and mict mutant lines were analyzed. For cutin analysis, fully expanded leaves and fruits were delipidated in a $10 \mathrm{ml}$ mixture of methanol-chloroform (1:1, v:v) for 2 weeks (solvent changed daily), after which the material was dried, weighed, and used for analysis ${ }^{44}$.

\section{Statistical analyses}

All data are expressed as mean value \pm standard deviation (SD) of biological replicates. Statistical significance of the treatment differences was assessed using the Student's $t$-test when only two components were compared. In addition, $P$ values are from one-way ANOVA (Tukey's multiple comparisons test, $\alpha=0.05)$. GraphPad Prism version 8.2.1 for Windows (GraphPad Software, La Jolla, CA, USA) was used for the statistical analyses.

\section{Acknowledgements}

We thank the reviewers for critically reading the manuscript. This work was supported by the National Key R\&D Program of China (Grant No. 2018YFD0100701), the National Natural Science Foundation of China (31471156), the Shanghai Agriculture Applied Technology Development Program of China (G2015060402), and Shanghai Jiao Tong University "Life Science" Interdisciplinary Research of China (20ZD-02).

\section{Author details \\ ${ }^{1}$ School of Agriculture and Biology, Shanghai Jiao Tong University, Shanghai 200240, China. ${ }^{2}$ School of Design, Shanghai Jiao Tong University, Shanghai 200240, China. ${ }^{3}$ Hunan Vegetable Research Institute, Hunan Academy of Agriculture Sciences, Changsha 410125, China. ${ }^{4}$ Joint International Research Laboratory of Metabolic \& Developmental Sciences, School of Life Sciences and Biotechnology, Shanghai Jiao Tong University, Shanghai 200240, China. ${ }^{5}$ State Key Laboratory of Vegetable Germplasm Innovation, Tianjin 300384, China}

\section{Author contributions}

JS.P, G.W., J.P., and L.Z. conceived the project and designed the research. J.P. and L.Z. performed most of the experiments with help from G.C., H.W., Y.C., H.D., and J.Z.; J.P. and L.Z. analyzed the data and wrote the article with assistance from H.H., H.L., H.C., J.S., R.C., G.W., and JS.P.; J.P. and L.Z. supervised the project and completed the writing.
Conflict of interest

The authors declare that they have no conflict of interest.

Supplementary Information accompanies this paper at (https://doi.org/ 10.1038/s41438-020-00456-0).

Received: 11 August 2020 Revised: 10 October 2020 Accepted: 20 November 2020

Published online: 01 February 2021

\section{References}

1. Choi, Y. E. et al. Detoxification of cadmium in tobacco plants: formation and active excretion of crystals containing cadmium and calcium through trichomes. Planta 213, 45-50 (2010).

2. Werker, E. Trichome diversity and development. Adv. Botanical Res. 31, 1-35 (2000).

3. Schellmann, S. \& Hulskamp, M. Epidermal differentiation: trichomes in Arabidopsis as a model system. Int. J. Dev. Biol. 49, 579-584 (2005).

4. Jakoby, M. J. et al. Transcriptional profiling of mature Arabidopsis trichomes reveals that NOECK encodes the MIXTA-like transcriptional regulator MYB106. Plant Physiol. 148, 1583-1602 (2008).

5. Tian, N. et al. Overexpression of BraLTP2, a lipid transfer protein of Brassica napus, results in increased trichome density and altered concentration of secondary metabolites.Int. J. Mol. Sci. 19, 1733 (2018).

6. Li, L. et al. The tomato homolog of CORONATINE-INSENSITIVE1 is required for the maternal control of seed maturation, jasmonate-signaled defense responses, and glandular trichome development. Plant Cell 16, 126-143 (2004).

7. Kang, J. H., Shi, F., Jones, A. D., Marks, M. D. \& Howe, G. A. Distortion of trichome morphology by the hairless mutation of tomato affects leaf surface chemistry. J. Exp. Bot. 61, 1053-1064 (2010).

8. Kunst, L. \& Samuels, L. Plant cuticles shine: advances in wax biosynthesis and export. Curr. Opin. Plant Biol. 12, 721-727 (2009).

9. Li, F. et al. Identification of the wax ester synthase/acyl-coenzyme A: diacylglycerol acyltransferase WSD1 required for stem wax ester biosynthesis in Arabidopsis. Plant Physiol. 148, 97-107 (2008).

10. Bernard, A. et al. Reconstitution of plant alkane biosynthesis in yeast demonstrates that Arabidopsis ECERIFERUM1 and ECERIFERUM3 are core components of a very-long-chain alkane synthesis complex. Plant Cell 24, 3106-3118 (2012).

11. Hannoufa, A., McNevin, J. \& Lemieux, B. Epicuticular waxes of eceriferum mutants of Arabidopsis thaliana. Phytochemistry 33, 851-855 (1995).

12. Tresch, S., Heilmann, M., Christiansen, N., Looser, R. \& Grossmann, K. Inhibition of saturated very-long-chain fatty acid biosynthesis by mefluidide and perfluidone, selective inhibitors of 3-ketoacyl-CoA synthases. Phytochemistry 76, 162-171 (2012).

13. Pascal, S. et al. The Arabidopsis cer 26 mutant, like the cer 2 mutant, is specifically affected in the very long chain fatty acid elongation process. Plant J. 73, 733 (2013).

14. Borisjuk, N., Hrmova, M. \& Lopato, S. Transcriptional regulation of cuticle biosynthesis. Biotechnol. Adv. 32, 526-540 (2014).

15. Yan, T. et al. A novel HD-ZIP IV/MIXTA complex promotes glandular trichome initiation and cuticle development in Artemisia annua. N. Phytologist 218, 567-578 (2018).

16. Wang, Y.L. et al. Identification and mapping of Tril, a homeodomain-leucine zipper gene involved in multicellular trichome initiation in Cucumis sativus. Theor. Appl. Genet. 129, 305-316 (2016).

17. Zhao, J. L. et al. Micro-trichome as a class I homeodomain-leucine zipper gene regulates multicellular trichome development in Cucumis sativus. J. Integr. Plant Biol. 57, 925-935 (2015).

18. Damian, S. et al. STRING v10: protein-protein interaction networks, integrated over the tree of life.Nucleic Acids Res. 43, D447-D452 (2015).

19. Yang, $C$. et al. A regulatory gene induces trichome formation and embryo lethality in tomato. Proc. Natl Acad. Sci. 108, 11836-11841 (2011).

20. Yan, T. et al. HOMEODOMAIN PROTEIN 1 is required for jasmonate-mediated glandular trichome initiation in Artemisia annua. N. Phytologist 213, 1145-1155 (2016).

21. Yeats, T. H. \& Rose, J. K. C. The formation and function of plant cuticles. Plant Physiol. 163, 5-20 (2013). 
22. Lü, S. et al. Arabidopsis CER8 encodes LONG-CHAIN ACYL-COA SYNTHETASE 1 (LACS1) that has overlapping functions with LACS2 in plant wax and cutin synthesis. Plant J. 59, 553-564 (2009).

23. Wang, W. et al. Cucumber ECERIFERUM1 (CSCER1), which influences the cuticle properties and drought tolerance of cucumber, plays a key role in VLC alkanes biosynthesis. Plant Mol. Biol. 87, 219-233 (2015).

24. Greer, S. et al. The cytochrome P450 enzyme CYP96A15 is the midchain alkane hydroxylase responsible for formation of secondary alcohols and ketones in stem cuticular wax of Arabidopsis. Plant Physiol. 145, 653-667 (2007).

25. Fabre, G. et al. The ABCG transporter PEC1/ABCG32 is required for the formation of the developing leaf cuticle in Arabidopsis. N. Phytol. 209, 192-201 (2016).

26. Takeda, S., Iwasaki, A., Tatematsu, K. \& Okada, K. The half-size ABC transporter FOLDED PETALS 2/ABCG13 is involved in petal elongation through narrow spaces in Arabidopsis thaliana floral buds. Plants 3, 348-358 (2014).

27. Fiebig, A. et al. Alterations in CER6, a gene identical to CUT1, differentially affect long-chain lipid content on the surface of pollen and stems. Plant Cell 12, 2001-2008 (2000).

28. Oshima, Y. \& Mitsuda, N. The MIXTA-like transcription factor MYB16 is a major regulator of cuticle formation in vegetative organs. Plant Signal. Behav. 8, e26826 (2014).

29. Busta, L., Hegebarth, D., Kroc, E. \& Jetter, R. Changes in cuticular wax coverage and composition on developing Arabidopsis leaves are influenced by wax biosynthesis gene expression levels and trichome density. Planta $\mathbf{2 4 5}$ 297-311 (2017).

30. Marks, M. D., Wenger, J. P., Gilding, E., Jilk, R. \& Dixon, R. A. Transcriptome analysis of Arabidopsis wild-type and gl3-sst sim trichomes identifies four additional genes required for trichome development. Mol. Plant 2, 803-822 (2009).

31. Kuhn, B. M., Geisler, M., Bigler, L. \& Ringli, C. Flavonols accumulate asymmetrically and affect auxin transport in Arabidopsis. Plant Physiol. 156, 585-595 (2011).

32. Falcone Ferreyra, M. L. et al. Cloning and characterization of a UV-B-inducible maize flavonol synthase. Plant J. 62, 77-91 (2010).
33. Pollastri, S. \& Tattini, M. Flavonols: old compounds for old roles. Ann. Bot. 108 1225-1233 (2011).

34. Wang, W. et al. Cucumis sativus L. WAX2 plays a pivotal role in wax biosynthesis, influencing pollen fertility and plant biotic and abiotic stress responses.Plant Cell Physiol. 56, 1339-1354 (2015).

35. Pan, J. et al. Differential gene expression caused by the $\mathrm{F}$ and $\mathrm{M}$ loci provides insight into ethylene-mediated female flower differentiation in cucumber. Front. Plant Sci. 9, 1091 (2018).

36. Li, Z. et al. A putative positive feedback regulation mechanism in CsACS2 expression suggests a modified model for sex determination in cucumber (Cucumis sativus L.). J. Exp. Bot. 63, 4475-4484 (2012).

37. Wen, C. et al. CSTFL1 inhibits determinate growth and terminal flower formation through interaction with CsNOT2a in cucumber. Development 146 v180166 (2019).

38. Li, G. et al. Characterization of candidate class A, B and E floral homeotic genes from the perianthless basal angiosperm Chloranthus spicatus (Chloranthaceae). Dev. Genes Evol. 215, 651 (2005).

39. Chen, C. et al. Transcriptome profiling reveals roles of meristem regulators and polarity genes during fruit trichome development in cucumber (Cucumis sativus L.). J. Exp. Bot. 65, 4943-4958 (2014).

40. Huang, S. et al. The genome of the cucumber, Cucumis sativus L. Nat. Genet 41, 1275-1281 (2009).

41. Chen, M., Yan, T., Shen, Q., Lu, X. \& Tang, K. GLANDULAR TRICHOME-SPECIFIC WRKY 1 promotes artemisinin biosynthesis in Artemisia annua. N. Phytologist 214, 304 (2016).

42. Yang, S. et al. A CsMYB6-CsTRY modulule regulates fruit trichome initiation in cucumberJ. Exp. Bot. 69, 1887-1902 (2018).

43. $\mathrm{Hu}, \mathrm{C}$. et al. Identification of conserved and diverse metabolic shift of the stylar, intermediate and peduncular segments of cucumber fruit during development. Int. J. Mol. Sci. 19, 135 (2018).

44. Kurdyukov, S. et al. Genetic and biochemical evidence for involvement of HOTHEAD in the biosynthesis of long-chain a-, $\omega$-dicarboxylic fatty acids and formation of extracellular matrix. Planta 224, 315-329 (2006). 